\title{
Combination and QCD Analysis of the HERA Inclusive Cross Sections
}

\author{
Voica Radescu ${ }^{* \dagger}$ \\ Physikalisches Institut, University of Heidelberg \\ E-mail: voica@mail.desy.de
}

\begin{abstract}
A QCD fit analysis to the combined HERA-I inclusive deep inelastic cross sections measured by the H1 and ZEUS collaborations for $e^{ \pm} p$ scattering resulting into a competitive NLO PDF set, HERAPDF1.0 is presented. HERAPDF at NNLO fits are presented as well, resulting, however, in a worse description of the combined HERA data. In addition, a preliminary analysis including the HERA II measurements of lower proton-beam energies is performed. The effect of including the new data on the determination of HERA parton distribution functions is analysed, using fits similar to those performed for HERAPDF1.0. Some tension of the QCD fit with respect to the data is identified in the kinematic region of low $Q^{2}$ and low $\mathrm{x}$. Finally, the QCD fit analysis of the combined HERA-I inclusive deep inelastic cross sections has been extended to include combined HERA II measurements at high $Q^{2}$. The effect of including these data on the determination of parton distribution functions is analysed, resulting into HERAPDF1.5. The precision of the new PDFs at high-x is considerably improved, particularly in the valence sector.
\end{abstract}

35th International Conference of High Energy Physics - ICHEP2010,

July 22-28, 2010

Paris France

\footnotetext{
*Speaker.

${ }^{\dagger}$ On behalf of the $\mathrm{H} 1$ and ZEUS collaborations
} 


\section{Combined H1 and ZEUS Cross Section Measurements}

Deep inelastic scattering (DIS) at HERA has been crucial to the exploration of proton structure and quark-gluon interaction dynamics. The combination of the H1 and ZEUS data provides the most accurate measurements of DIS inclusive double differential cross-sections of neutral (NC) and charged current (CC) $e^{ \pm} p$ scattering over an extended kinematic range of $0.045<Q^{2}<$ $30000 \mathrm{GeV}^{2}$ and $6 \times 10^{-5}<x<0.65$. Therefore, these accurate measurements can be used as a sole input to the QCD analysis to determine the proton structure - parton distribution functions (PDFs) as described in the following sections, which can be used then for precise predictions for the LHC processes.

The combination of data uses the $\chi^{2}$ minimisation method and it takes into account the correlated systematic uncertainties for the H1 and ZEUS cross-section measurements [1]. This combination procedure is applied for the following scenarios: to the published inclusive deep inelastic cross sections measured by the $\mathrm{H} 1$ and ZEUS collaborations in CC and $\mathrm{NC}$ unpolarised $e p$ scattering at HERA during the period 1994-2000, termed HERA I; to the preliminary inclusive deep inelastic cross sections measured by the H1 and ZEUS collaborations in NC unpolarised $e p$ scattering at HERA during its last months of operation in 2007 with reduced proton beam of $E_{p}=460 \mathrm{GeV}$ and $E_{p}=575 \mathrm{GeV}$ which provides additional PDF constraint in the low $Q^{2}$, low $x$ region; and to the preliminary inclusive deep inelastic NC and CC cross sections at high $Q^{2}$ measured by the $\mathrm{H} 1$ and ZEUS collaborations at HERA during the whole period which considerably improve PDF uncertainties at high- $x$ as described in these proceedings.

\section{QCD Analysis settings}

The above combined data is used as a sole input into a QCD fit analysis to extract the proton's PDFs. The HERA data have a minimum invariant mass of the hadronic system, $W$, of $15 \mathrm{GeV}$ and a maximum $x$ of 0.65 , such that they are in a kinematic region where there is no sensitivity to target mass and large- $x$ higher-twist contributions. In addition, to restrain to the region where perturbative QCD is valid, only data above $Q_{\min }^{2}=3.5 \mathrm{GeV}^{2}$ is used in the central fit.

The fit procedure consists first in parametrising PDFs at a starting scale $Q_{0}^{2}=1.9 \mathrm{GeV}^{2}$, chosen to be below the charm mass threshold. The parametrised PDFs are the valence distributions $x u_{v}$ and $x d_{v}$, the gluon distribution $x g$, and the $u$-type and $d$-type $x \bar{U}, x \bar{D}$, where $x \bar{U}=x \bar{u}, x \bar{D}=x \bar{d}+x \bar{s}$. The following standard functional form is used to parametrise them

$$
x f(x)=A x^{B}(1-x)^{C}\left(1+D x+E x^{2}\right),
$$

where the normalisation parameters, $A_{u v}, A_{d v}, A_{g}$, are constrained by the QCD sum-rules. The $B$ parameters $B_{\bar{U}}$ and $B_{\bar{D}}$ are set equal, $B_{\bar{U}}=B_{\bar{D}}$, such that there is a single $B$ parameter for the sea distributions. The strange quark distribution is already present at the starting scale and it is assumed here that $x \bar{s}=f_{s} x \bar{D}$ at $Q_{0}^{2}$. The strange fraction is chosen to be $f_{s}=0.31$ which is consistent with determinations of this fraction using neutrino induced di-muon production. In addition, to ensure that $x \bar{u} \rightarrow x \bar{d}$ as $x \rightarrow 0, A_{\bar{U}}=A_{\bar{D}}\left(1-f_{s}\right)$. The $D$ and $E$ are introduced one by one until further improvement in $\chi^{2}$ is found. The best fit results in a total of 10 free parameters.

The PDFs are then evolved using DGLAP evolution equations [5]. at NLO and NNLO in the $\overline{M S}$ scheme with the renormalisation and factorisation scales set to $Q^{2}$. The QCD predictions 
for the structure functions are obtained by convoluting the PDFs with the calculable coefficient functions taking into account mass effect for the heavy quarks based on the general mass variable flavour scheme [4].

The uncertainties at HERA are classified in three categories: experimental, model, and parametrisation uncertainties. The consistency of the input data set and its small systematic uncertainties enable us to calculate the experimental uncertainties on the PDFs using the $\chi^{2}$ tolerance $\Delta \chi^{2}=1$. The model uncertainties are evaluated by varying the input assumptions, which are the variation of the starting scale and of the $Q_{\min }^{2}$, the variations of the heavy quark masses which are set to the standard values of $m_{c}=1.4 \mathrm{GeV}$ and $m_{b}=4.75 \mathrm{GeV}$ for the central fit, and the variation of $f_{s}$. The parametrisation uncertainty is estimated as an envelope which is formed as a maximal deviation at each $x$ value from the central fit of 10 parameter fits with $D$ and $E$ non-zero from Equation 2.1.

\section{Results and Comparisons}

The NLO QCD analysis has been performed first to the final HERA I data resulting into HERAPDF1.0 which has been published [2] and it will be used as a reference for the new studies. The NNLO fit results have been also performed using the same scheme as used for MSTW PDF sets, for different values of the strong coupling, $\alpha_{s}\left(M_{Z}\right)=0.1176$ and $\alpha_{s}\left(M_{Z}\right)=0.1145$ [6]. Fit results are shown in Figure 1. However, the NNLO fits do not bring improvement in terms of the fit quality with respect to NLO fits (worse by about 65 and 50 units of $\chi^{2}$, respectively) for both $\alpha_{S}$ cases, with a preference for lower value of the strong coupling at NNLO.

The inclusion of the new preliminary data at low proton beam energy in the HERA QCD fits results in PDF distributions that agree well with the HERAPDF1.0, as shown in Figure 1. However, a large sensitivity has been observed when the variation of the kinematic cut has been studied, i.e. $Q_{\min }^{2}>5 \mathrm{GeV}^{2}$, which yielded a different PDF shape for the gluon distribution with respect to the the central fit which uses $Q_{\min }^{2}>3.5 \mathrm{GeV}^{2}$.

The QCD fit analysis of the combined HERA-I inclusive deep inelastic cross sections has been extended to include combined HERA II measurements at high $Q^{2}$ resulting into HERAPDF1.5 [6]. Figure 2 shows that the precision of the PDFs at high- $x$ is considerably improved, not only for the experimental uncertainties, but also for the parametrisation uncertainty - particularly in the valence sector, when compared to HERAPDF1.0. This leads to more precise predictions for the LHC process.

The predictions based on HERAPDFs from the DIS process agree well with the Tevatron jet production, $Z$ and $W$ cross sections from the $p \bar{p}$ process and provide a competitive prediction for the LHC $p p$ processes.

\section{References}

[1] H1 Collaboration, F. Aaron et al. Eur.Phys.J.C64 (2009) 561, arXiv 0904.0929.

[2] H1 Collaboration, ZEUS Collaboration, F. Aaron et al. , JHEP 1001, 109 (2010), arXiv:0911.0884.

[3] C. Amsler et al. (Particle Data Group), Phys. Lett. B667, (2008).

[4] R. S. Thorne code, revised in 2008.

[5] QCDNUM package, M. Botje, (2010), arXiv:1005.1481, http://www.nikef.nl/h24/qcdnum/index.html

[6] The LHAPDF grid files are located at https://www.desy.de/h1zeus/combined_results/index.php?do=proton_structure 

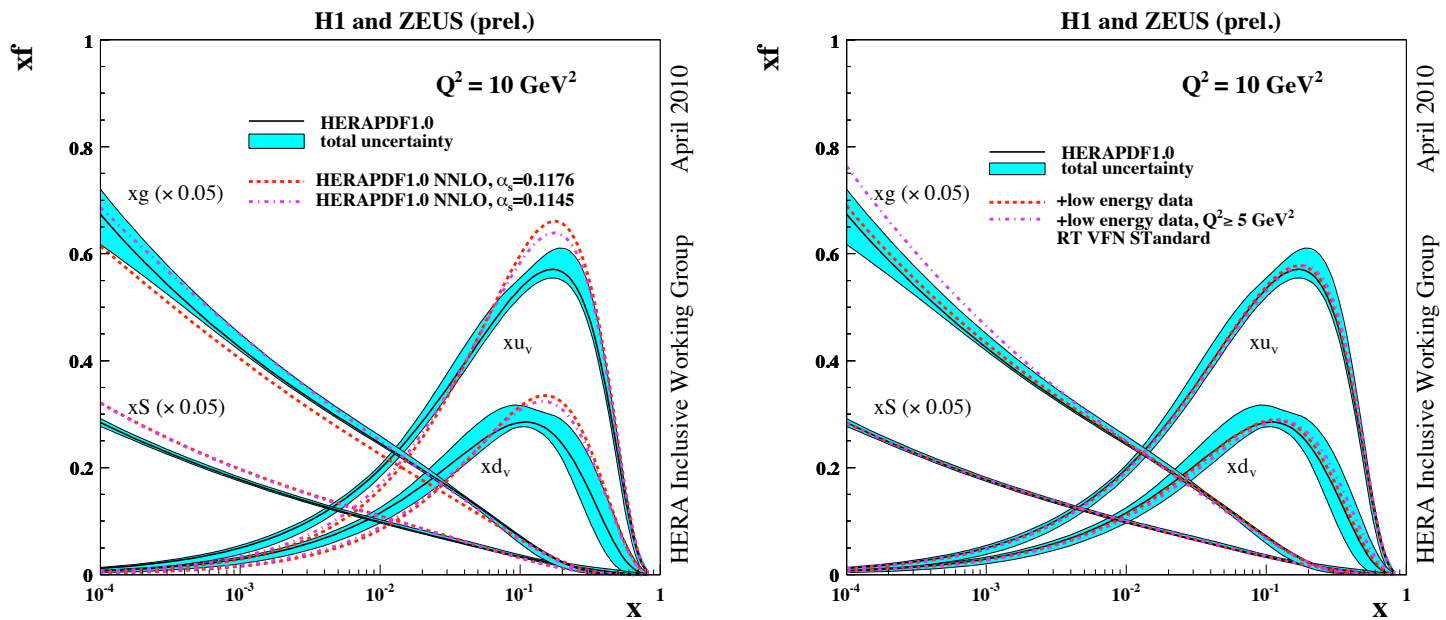

Figure 1: Figure shows on the left hand side the summary plot at the $Q^{2}=10 \mathrm{GeV}^{2}$ with gluon, sea (which are scaled by a factor of 0.05) and the valence distributions for HERAPDF1.0 at NLO (band) compared illustratively to NNLO fits using $\alpha_{S}\left(M_{Z}\right)=0.1145$ (dashed) and $\alpha_{S}\left(M_{Z}\right)=0.1176$ (dotted) lines. On the right hand side, it is shown the comparison between the HERAPDF1.0 (band) based on HERA I data and fits including the HERA II of lower energy proton beams with the kinematic cut variation $Q^{2} \geq 3.5$ (dotted) and $Q^{2} \geq 5.0$ (dashed).
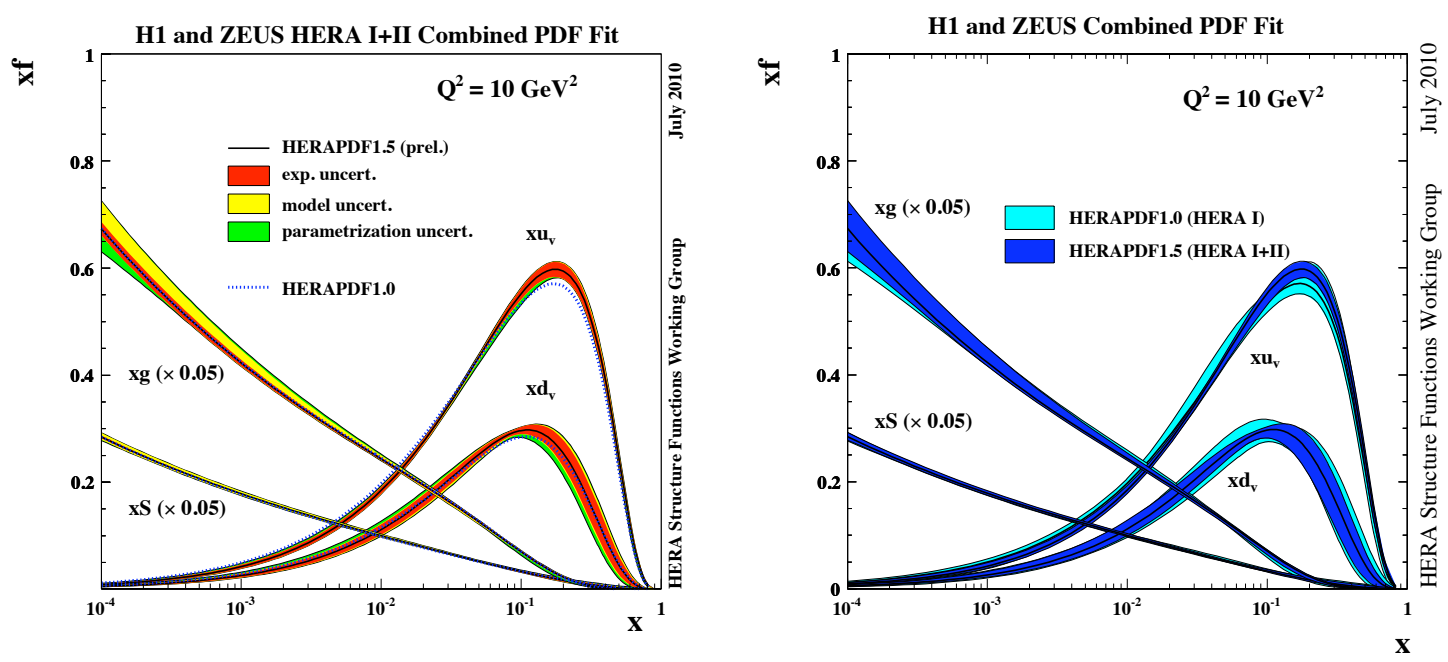

Figure 2: Figure shows on the left hand side the summary plot for the HERAPDF1.5 at the $Q^{2}=10 \mathrm{GeV}^{2}$ with gluon, sea (which are scaled by a factor of 0.05 ) and the valence distributions. The errors include the experimental (red), model (yellow) and the PDF parametrisation (green) uncertainties. On the right hand side, it is shown the comparison between the HERAPDF1.0 (light color) based on HERA I data and HERAPDF1.5 (dark color) based on HERA I and II data, using total uncertainty band at $Q^{2}=10 \mathrm{GeV}^{2}$. 